Critique de l'expérimentation: paradigmes politiques et technologies gouvernementales

\title{
Des expérimentations démocratiques en tension. L'œuvre des citoyens dans le travail politique des bifurcations
}

Francis Chateauraynaud

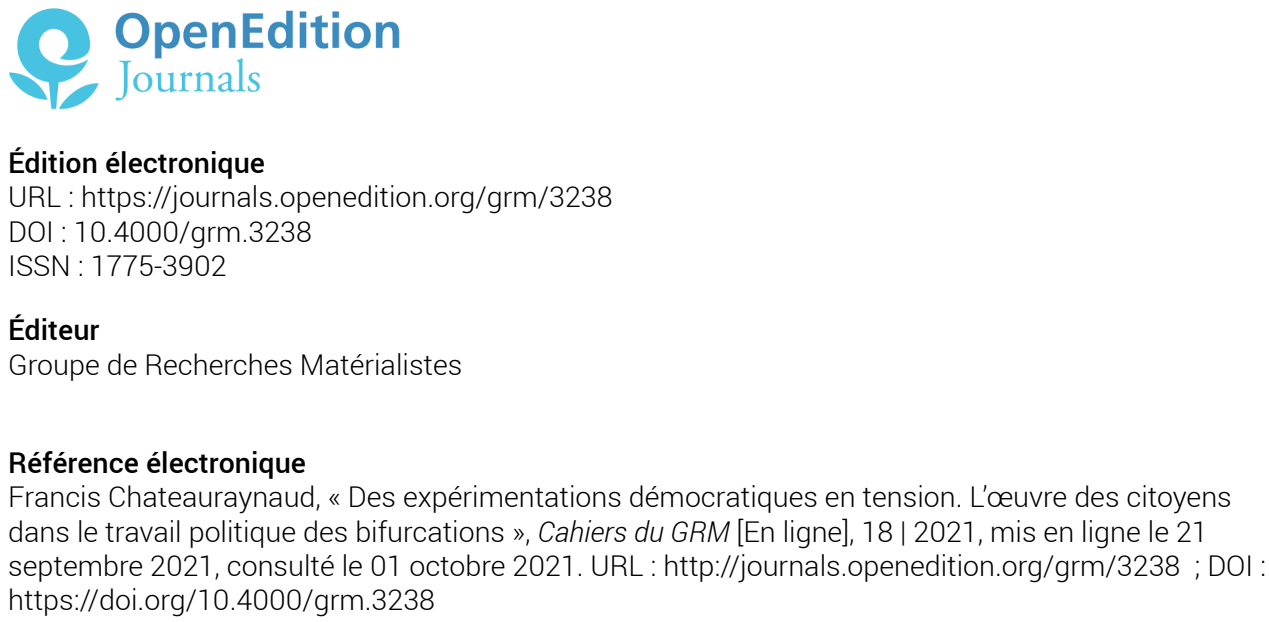

Ce document a été généré automatiquement le 1 octobre 2021.

(c) GRM - Association 


\title{
Des expérimentations démocratiques en tension. L'œuvre des citoyens dans le travail politique des bifurcations
}

\author{
Francis Chateauraynaud
}

1 «La politique, c'est la réunion, le lieu où les gens du peuple se parlent à eux-mêmes, sans recettes préétablies et choisissent de présenter leurs propositions, les traduisent en mots d'ordre. C'est la seule façon pour le peuple de se servir lui-même et construire sa politique. Le militant, c'est l'artisan de ce travail minutieux. »Cette formule, lancée par un internaute en juin 2021 sur le blog d'un média critique, repose sur un régime argumentatif utilisé depuis longtemps dans les arènes publiques. Toutes sortes d'auteurs-acteurs s'efforcent de rappeler ce que doit être une démocratie face à des décisions et des pratiques politiques qu'ils jugent illégitimes, autoritaires, déviantes ou catastrophiques. C'est d'autant plus vif que, depuis plusieurs décennies, le diagnostic de « crise de la représentation politique » fait partie des lieux communs les plus partagés par les élites politiques, qui manquent cruellement d'imagination lorsqu'il s'agit de refonder ladite démocratie ${ }^{1}$. Bien que l'abstentionnisme électoral ne soit qu'un aspect du problème, âprement discuté par les experts, la surenchère interprétative à laquelle ont donné lieu les élections régionales de juin 2021 a encore renforcé cette propension.

2 En termes d'analyse discursive, la démocratie donne lieu à une quantité astronomique d'énoncés, des plus théoriques aux plus évasifs, révélant malgré tout bien des tensions et des contradictions. Car le diagnostic de crise ne va pas sans paradoxe : parallèlement, ou plutôt de manière orthogonale à la politique instituée, de nombreux processus se déploient, détournant ou réinventant les formes d'expression, de délibération et d'action politique. Une partie de ces processus reçoit la qualification d'« expérimentation démocratique». Mais de quoi s'agit-il? Cette notion d'expérimentation s'inspire-t-elle des sciences expérimentales? Constitue-t-elle un contrepoint au mouvement de l'économie expérimentale ${ }^{2}$ ? N'est-ce pas simplement 
une autre façon de désigner toute mise à l'épreuve, tout essai ou innovation engageant la participation active de citoyen-ne-s ? Ou plus formellement, ce qu'on appelle une synecdoque généralisante, permettant de ramasser, dans une formule, une multitude d'expériences collectives? Cela dit, entre l'expérience et l'expérimentation se glisse une différence d'agentivité : dans les usages ordinaires, l'expérience désigne souvent des événements subis sans intentionnalité préalable, tandis que l'expérimentation implique a minima un dispositif intentionnel, liant une visée commune et une

organisation collective. En réalité, le pragmatisme a montré que toute expérience est une forme d'épreuve dans laquelle se révèlent des entités et des relations, et qu'elle engage intensément les personnes, rarement passives, au moins dans l'interprétation et l'analyse réflexive qu'elles font des événements ${ }^{3}$. Mais l'idée d'expérimentation démocratique engage une autre logique d'action et un autre concept d'expérience, ce qui conduit à la question suivante : $y$ a-t-il nécessairement un ou des organisateurs, des leaders ou des pilotes à bord, occupant la place de l'expérimentateur ou s'agit-il plutôt d'un processus ouvert, liant une auto-institution des règles de fonctionnement, une équitable distribution des prises de parole et des tâches, ainsi qu'une forte dimension d'improvisation et d'invention collective?

\section{Le pragmatisme et la sociologie face aux questions démocratiques}

3 La présente contribution ne peut réécrire la genèse des dispositifs "participatifs » ${ }^{4}$. Il s'agit plutôt d'examiner quelques-unes des tensions, des contraintes et des ressorts auxquels font face des personnes ordinaires lorsqu'elles tentent de peser sur un processus de délibération et de décision publique. L'exemple récent de la convention citoyenne pour le climat est sans doute l'arbre qui cache le mieux la forêt: lors de son lancement, cette procédure a fait l'objet de critiques, notamment de plusieurs ONG, quant à sa pertinence devant l'urgence écologique. La manière dont les gouvernants, suivis par les parlementaires, ont reformulé, détricoté ou écarté la plupart des 150 propositions élaborées par les groupes de citoyens tirés au sort ${ }^{5}$, semble donner raison ex post aux sceptiques. Mais cette expérience collective, bien que très contrainte au plan procédural, n'a pas été inutile sur le plan politique.

4 À partir de leur colère devant la manière dont les choses se sont passées, les dits citoyen-n-es ont créé une association dans le but de prolonger le travail collectif entrepris autour de leurs propositions et de briser la logique purement agrégative associée au tirage au sort d'individus supposés anonymes et voués à ne plus interagir après la fin de la procédure 6 .

5 Si la convention citoyenne était bien conçue par ses promoteurs comme une "expérimentation démocratique», soutenue par la montée en puissance de la référence au tirage au sort ${ }^{7}$, les tensions et les contradictions qu'elle a rendues manifestes dans l'exercice du pouvoir ont produit des conséquences sur plusieurs lignes de transformation liées aux enjeux climatiques, du local à l'international, tout en nourrissant des actions juridiques à travers lesquelles l'inaction de l'Etat est de plus en plus mise en cause ${ }^{8}$. Cette dimension transformative des expériences collectives mérite d'être prise au sérieux : comment des dispositifs formalisés, engageant des acteurs pris dans des cadres très contraignants, peuvent donner lieu, non seulement à un 
renouvellement des appuis critiques ${ }^{9}$, mais aussi et surtout à des détournements, des inventions ou de véritables subversions.

6 Pour saisir les transformations à l'œuvre aux points de jonction, et de friction, de trois ensembles formés par les arènes de débat, les sciences participatives et les modes de protestation, la sociologie contemporaine est conduite à réinvestir des questions et des méthodes proposées au début du XXème siècle par les philosophes pragmatistes et leurs nombreux partenaires au cœur des sciences sociales naissantes ${ }^{10}$. Ce retour aux sources du pragmatisme ne s'est pas effectué sans quelques déplacements épistémiques et sans une reformulation conceptuelle liée à la complexité particulière des processus contemporains ${ }^{11}$. Pour les lecteurs éloignés de ce champ de recherche, il convient de distinguer deux mouvements souvent confondus: d'un côté, il y a les sociologies pragmatiques à la française, issues à la fin des années 1980 de la sociologie des cités de Luc Boltanski et Laurent Thévenot, la théorie de l'acteur-réseau de Bruno Latour et Michel Callon, ainsi que les approches inspirées par l'ethnométhodologie et les théories de l'action située autour de chercheurs comme Louis Quéré, Patrick Pharo, Nicolas Dodier ou Bernard Conein; de l'autre côté, bien qu'il soit possible de traverser à gué par temps calme, se déploie un pragmatisme sociologique lui-même composite, qui a pour motif de rassemblement la lecture ou relecture des pères fondateurs du pragmatisme américain, Pierce, James, Dewey et Mead ${ }^{12}$.

7 Quel est, en deux mots, l'enjeu d'une réappropriation des philosophies pragmatistes par la sociologie contemporaine? Les transformations politiques des vingt dernières années ont rendu définitivement caduque la version hiérarchique, asymétrique et forcément objectiviste des compétences cognitives et politiques des personnes ordinaires, notamment dans leurs interactions multiples avec les groupes, réseaux et autres collectifs qu'elles initient, fréquentent, réinventent et investissent ${ }^{13}$. Alors que dans les années 1980-1990, l'attribution de fortes capacités réflexives, techniques et critiques aux acteurs était l'apanage des courants sociologiques contestant les théories hégémoniques du pouvoir, soit, en France, outre la tradition marxiste, Foucault, Bourdieu et Crozier. Mais la multiplication des expériences collectives, notamment à partir des mouvements de malades contre le Sida ${ }^{14}$, a bouleversé le programme des sciences sociales - du moins celles qui pratiquent intensément les enquêtes de terrain : le constat de départ n'est plus tributaire de la vieille épistémologie de la rupture avec le sens commun ; il part du fait que les enquêteurs professionnels entrent en interactions continues avec d'autres enquêteurs dont les capacités de réflexion et d'action sont souvent très aiguisées, plus agiles et aisément transportables d'un champ d'expérience à un autre. Il faut dire qu'elles sont délestées des contraintes académiques qui ont tendance à stériliser l'expression directe de toute expérience vivante ${ }^{15}$. Pour éviter le double piège d'une mise à distance objectivante et d'une adhésion sans recul vis-à-vis des causes et des doctrines d'acteurs, le pragmatisme sociologique invite à privilégier la construction d'espaces de variation permettant, sur un mode inclusif, de suivre au plus près les processus tout en se dotant d'une multiplicité d'appuis extérieurs.

8 Le pragmatisme sociologique a eu très tôt affaire à l'émergence de processus collectifs prenant de biais, ou de court, les procédures instituées. La participation volontaire et délibérée de citoyen-ne-s à des assemblées, des débats publics, des expériences délibératives ou des actions collectives n'est pas une invention récente et a une très longue histoire. Des événements marquants ressurgissent régulièrement dans les arènes publiques, à l'occasion de mouvements sociaux (avec la référence aux 
occupations de places dans le mouvement Nuit Debout puis par une partie des Gilets Jaunes ${ }^{16}$ ) ou lors de commémorations (comme les 150 ans de la Commune au printemps 2021). En sociologie politique, on se réfère surtout à l'imposante bifurcation qui s'est produite au cours des années 1970, tant dans les répertoires d'action et d'argumentation liés aux nouvelles formes de lutte, que dans les innovations procédurales, les formes d'enquête et de délibération collective. La littérature est immense sur les expériences politiques des années 1970, de LIP au Larzac, en passant par une multitude de terrains de lutte et de conflit. Dans leur ouvrage, Lutte urbaine. Participation et démocratie d'interpellation à l'Alma-Gare, Paula Cossart et Julien Talpin refont le récit d'une lutte urbaine mythique, menée dans un quartier pauvre de Roubaix, l'Alma-Gare ${ }^{17}$. Ils montrent comment, en s'organisant contre la destruction de leurs logements, les habitants ont redessiné les plans d'un quartier selon leurs aspirations, transformant leur lutte en expérimentation d'autogestion visant à " organiser la vie sociale différemment », tout en témoignant d'« un réel pouvoir d'agir des classes populaires ». Avec la fin de la dynamique collective initiale, le quartier a progressivement glissé vers la relégation sociale, mais les auteurs, férus d'empowerment, plaident pour «l'avènement d'une démocratie participative exigeante qui ne soit pas un nouvel outil de domination des plus faibles ».

9 Il n'est guère possible de déployer complètement l'espace de variation, à la fois critique et pratique, qui sous-tend l'idée d'expérimentation démocratique. Entre les assemblées citoyennes qui émergent sur le terrain des luttes sociales ou écologiques et les dispositifs institués par le haut, entre les formules de démocratie locale, de plus en plus inspirées par le municipalisme libertaire et les débats publics liés à des enjeux, souvent conflictuels, d'aménagement ou d'infrastructure, le répertoire des cas de figure est immense. Pour en dégager les grands lignes, on peut s'appuyer sur trois séries de travaux et de discussion, afin d'en proposer une lecture analytique et (re)constructive ${ }^{18}$ : les apports du pragmatisme sociologique à l'analyse des contradictions politiques des sociétés contemporaines ; les recherches sur les processus participatifs et les formes de contestation associées; les formes de scénarisation des futurs prises en étau entre l'urgence écologique et sociale (crise climatique et explosion des inégalités) et la répression des alternatives par la montée de nouvelles formes d'autoritarisme ${ }^{19}$.

Pour convaincre que la casuistique est désormais plus qu'étoffée, tentons malgré tout une brève énumération de quelques moments forts de l'histoire politique récente dans lesquels la question de l'expérience démocratique, et de son organisation sous forme d'expérimentation collective, s'est posée. En indiquant seulement les processus que l'on a pu examiner en détail, lors d'enquêtes ou de séminaires, on peut se déplacer de la conférence de citoyens sur les OGM (1998) à la convention citoyenne pour le climat (2020), du débat numérique sur la carte d'identité électronique (2003) aux formes d'expression du mouvement Nuit Debout (2016), des multiples initiatives liées au référendum sur la constitution européenne en 2005 à l'installation d'une municipalité citoyenne à Sivens (2014), du débat public organisé par la CNDP ${ }^{20}$ sur le nucléaire (2005) au contre-sommet de la Terre à Rio en 2012. Les jeux d'acteurs, les enjeux, les procédures, les échelles sont très variables mais dans tous les cas se déploie, à travers des dispositifs d'expression collective, un intense travail critique et argumentatif. Ce travail politique est porté par des personnes et des groupes dotés de titres et de compétences différentes mais qui ont en commun d'être déterminés à faire bouger les lignes, à pousser des alternatives, contester l'évidence du système économique 
dominant et de proposer de nouvelles perspectives pour le futur, proche et lointain. A ce premier ensemble, intensément étudiés par les sciences sociales contemporaines, il faut encore ajouter deux autres univers dans lesquels la notion d'expérimentation démocratique est pleinement dotée de senspar les acteurs eux-mêmes: les innombrables expériences de recherche participative, en particulier dans les sciences environnementales ${ }^{21}$; les multiples performances de désobéissance civique qui ont marqué les mobilisations collectives - de l'aide aux étrangers en situation irrégulière au mouvement des décrocheurs - et ont renouvelé les modalités d'expression, d'argumentation et d'action.

\section{L'expérimentation démocratique comme mise à l'épreuve axiologique, épistémique et ontologique}

11 Le pragmatisme s'est démarqué des philosophies classiques, qui avaient investi la question de la rationalité et de la connaissance sans remettre en cause la dichotomie sujet/objet, ainsi que des grandes théories critiques reposant sur la double idée d'aliénation et de réification justifiant une logique de dévoilement. Dans le mouvement pragmatiste, il s'agit d'abord de lier la question de l'expérience du monde à sa mise à l'épreuve. Celle-ci est saisie comme un processus ouvert dans lequel se joue aussi bien la formation de publics, la production de faits à travers les enquêtes et la mise en discussion des possibles selon les modes de valuation développés par les personnes et les groupes. L'attention à la formation des problèmes publics conduit ainsi à révéler un sens ordinaire de l'expérimentation, nécessaire à la formation de citoyen-ne-s en démocratie. Daniel Cefaï a proposé une archéologie de l'idée d'expérimentation démocratique, en explorant comment Dewey et Mead ont cherché à rapprocher les formes d'expérimentation développées dans les laboratoires scientifiques et les expériences des laboratoires naturels constitués par les multiples situations de la vie sociale $^{22}$. Cette remise en histoire du pragmatisme fait apparaître l'importance précoce de l'entrée par les situations d'épreuve (test situations), et leur lien insécable avec les affaires civiques et politiques. Le rapprochement de l'expérimentation scientifique et de la mise à l'épreuve en monde ouvert n'est pas fortuite : « Il s'agit dans tous les cas, écrit Cefaï, de tester en pratique (et pas simplement en discours) des hypothèses de travail (et non pas de simples professions de foi) pour en déterminer un certain type de validité, eu égard à leurs conséquences ». Et le conséquentialisme, on le sait, est un des ressorts majeurs du raisonnement et de l'enquête pragmatiste.

12 Essayons de préciser, à partir des leçons fournies par les expériences collectives contemporaines, la relation foncière entre expérimentation et raisonnement par les conséquences. En cherchant, dans la casuistique évoquée plus haut, les trames communes aux différents mouvements opérés par les acteurs dans des contextes spécifiques, dans lesquels opère à des degrés divers une critique générale du système capitaliste, trois dimensions, ou trois axes, s'imposent sur lesquels opèrent les mises à l'épreuve et les boucles réflexives effectuées par les acteurs. Il est important de noter dès à présent que la critique n'est pas évincée du pragmatisme puisqu'elle est avant tout restituée aux acteurs: ses ressorts ne sont plus le monopole de clercs ou de professions intellectuelles, mais sont rendus manifestes à travers l'énonciation, l'évaluation et la hiérarchisation des conséquences - qui en pratique accompagnent toute décision de passage à l'action ${ }^{23}$. 
13 Le premier axe oppose les principes de jugement déposés dans les institutions, dont dépend l'ordre politique existant, et les formes de valuation, d'interprétation et de mise en récit produites au cœur des expériences, dans les milieux et les dispositifs. L'expérimentation consiste alors à tester toutes les mises en variation critique d'ordre axiologique et à en tirer toutes les conséquences : faut-il autoriser ou interdire ceci ou cela ; doit-on normaliser ou valoriser telle ou telle pratique etc. Le deuxième axe croise le premier, ou plutôt s'enroule autour à partir de plusieurs nœuds : il met en tension des connaissances stabilisées, déposées dans des instances scientifiques reconnues, et des savoirs émergents ou ancrés dans des champs d'expérience spécifiques ou locaux et ne disposant pas immédiatement de la légitimité épistémique qui se déploie à l'autre bout du continuum. Ce deuxième axe est donc celui de la mise à l'épreuve des connaissances et des savoirs, mais aussi des méthodes. La notion d'expérimentation est centrale dans les querelles de méthode, et le pragmatisme en donne une version différente de celle du rationalisme, en commençant par revoir le statut de l'expérimentateur. Un troisième axe, que l'on peut qualifier d'ontologique, concerne les catégories d'entités et de relations auxquelles se réfèrent couramment les acteurs : des plus communément admises, assurant un minimum de sens commun de la réalitée ${ }^{24}$, aux formes de vie et mode d'existence les plus insolites, il permet d'ouvrir le raisonnement et l'enquête à toutes les variations imaginaires - les hypothèses au cœur des mises à l'épreuve se révélant bien pauvres sans le secours de l'imagination ${ }^{25}$. Sur cet axe ontologique, l'enjeu de l'expérimentation concerne directement la possibilité-même de formes d'existence, de leur visibilité et de leur tangibilité dans la vie pratique. Ce troisième axe a aussi pour caractéristique de s'enrouler de façon non-linéaire aux deux premiers - à ceci près que, lorsqu'une forme de vie ou un mode d'existence est contesté ou nié, le niveau de conflictualité et de violence augmente très vite et sans garde-fou ${ }^{26}$.

L'engouement contemporain pour la coproduction des savoirs n'est guère soutenable sans intégrer le différend, le conflit ou le rapport de forces dans les analyses, y compris dans ce qui touche aux techniques. Expérimenter en démocratie, c'est à la fois prendre le risque de la conflictualité, de la nourrir et de rendre impossible un accord général sur une question ou un "problème public ", et remettre en politique des dispositifs présentés comme neutres ou pensés jusqu'alors dans une pure logique d'efficacité technique - c'est clairement le cas des outils numériques aujourd'hui ${ }^{27}$. La reconnaissance de la possibilité permanente du désaccord, de la défiance et du conflit, est adossée au pluralisme, maxime décisive du pragmatisme, y compris dans les questions épistémologiques et méthodologique. C'est particulièrement décisif lorsque des controverses de fond ne sont pas tranchées et que perdure la question du statut et de la portée d'une ou plusieurs méthodes. La longue crise du Covid-19 nous a fourni de multiples exemples de ce type de tension, depuis les querelles de chapelles biomédicales jusqu'aux polémiques autour du complotisme. La résolution des différends repose souvent, outre la fatigue des combattants, sur un accord quant à la manière de mener les enquêtes et donc sur des questions de méthode ${ }^{28}$.

\section{La méthode comme élaboration collective de prises communes}

L'accès au processus qui sous-tend toute méthode, qu'elle engage des scientifiques ou des personnes ordinaires, est ce qui assure la portée d'une expérimentation dans sa 
double dimension cognitive et politique. Dans la version démocratique de l'expérimentation, la place de l'expérimentateur n'est plus monopolisée, figée, adossée à un statut : elle est empruntée tour à tour par les membres du collectif d'enquêteurs ou de débatteurs, qui assurent la circulation des savoirs et des techniques de mise à l'épreuve ${ }^{29}$. Cela fait une énorme différence avec le « scientisme » qui assigne d'emblée aux «profanes » ou aux «non-experts» des places subalternes dans les processus de démonstration. Autrement dit, au-delà de la mode qui consiste à invoquer la "coproduction" ou la "co-construction", l'expérimentation démocratique a pour vocation de rendre interchangeables les rôles et les places, en cultivant des formes d'apprentissage, de transfert de savoirs et de compétences qui permettent de distribuer les prises individuelles et de faire émerger des prises collectives ${ }^{30}$. Dans de nombreux cas de figure, la production commune des méthodes pose néanmoins des problèmes de validité et de légitimité, car elle repose sur une dynamique collective qui ne va pas de soi, toute mise en commun des prises supposant une réduction maximale des asymétries et donc le contrôle par les membres des jeux de pouvoir et d'influence.

Un processus d'enquête peut être qualifié d'expérimentation démocratique dès lors que la définition de l'objet et de la méthode donne lieu à une délibération ouverte. Si les normes et les standards de la recherche scientifique publique sont souvent réinvestis pas les acteurs, il s'agit moins d'une forme de déférence épistémique pour l'autorité de la science que le résultat d'une négociation des termes et des critères susceptibles d'une appréciation commune, dans laquelle la connaissance pratique des milieux et des dispositifs est tout aussi primordiale que les canons épistémologiques. Parmi les exemples particulièrement réussis, on peut citer le cas du Réseau Semences Paysannes (RSP). Derrière l'opposition à l'agroindustrie et à son impérialisme méthodologique sur les pratiques agricoles, exprimée notamment lors du conflit des OGM, les membres du RSP ont pu échanger, expérimenter, développer des savoirs à partir de dispositifs inspirés du monde de l'open source, allant jusqu'à pratiquer une forme de sélection génétique participative ${ }^{31}$. Un autre exemple est fourni par les capteurs-citoyens qui ont été capables, notamment en matière de pollution de l'air, de défier les métrologies dominantes et d'inventer d'autres protocoles au plus près des expériences, sans doctrine définitive, en adoptant un principe de mise en discussion permanente des mesures, des sites visés, des modes d'étalonnage et des points de comparaison ${ }^{32}$.

17 L'expérimentation démocratique se distingue néanmoins des formes de contreexpertise, comme celles qui ont marqué les scènes publiques du risque technologique et environnemental depuis les années 1990, avec par exemple le CRII-RAD, le CRIIGEN ou PRIARTEM, collectifs qui se sont imposés, avec des degrés inégaux de légitimité, respectivement dans le champ des mesures de radioactivité, dans celui de la veille critique sur les biotechnologies ou encore à propos des effets sanitaires des ondes électromagnétiques. L'usage du qualificatif «citoyen» ou de la formule «science citoyenne » est souvent excessif dans la mesure où la maitrise des instruments reste confinée entre les mains d'un groupe réduit d'acteurs, même s'ils interviennent au service de riverains ou de collectifs de victimes ${ }^{33}$. Il reste que la multiplicité des expériences rend visible un continuum allant du simple décalage méthodologique motivé par une défiance vis-à-vis de l'expertise scientifique officielle, jugée trop dépendante des pouvoirs, jusqu'à la reconfiguration complète du monde vécu, des techniques et du répertoire d'action utilisés pour le faire exister ${ }^{34}$. 


\section{L'institutionnalisation des expériences participatives et ses critiques}

18 Est-ce que l'ensemble des expériences collectives observées par la sociologie contemporaine sont fongibles dans le pragmatisme classique? Dans sa Logique d'enquête, John Dewey soutient l'idée d'un canon de la méthode expérimentale tout en le déclinant dans plusieurs domaines de façon à relier production des savoirs qui importent et construction des publics concernés ${ }^{35}$. Attaché à mettre en évidence le caractère processuel de l'enquête et à détacher l'émergence des publics de positions établies par avance, il reste néanmoins peu loquace sur les controverses et les disputes qui traversent nécessairement les collectifs en formation, notamment quant à la radicalité des questions et des hypothèses. Or, comme indiqué plus haut, une véritable expérimentation démocratique peut faire apparaître des points de friction ou de disjonction susceptible de re-diviser ou re-fragmenter les publics - ce qui s'observe dans la plupart des processus de mobilisation suivis à ce jour ${ }^{36}$. Une des lignes de partage les plus visibles est celle qui oppose les acteurs qui acceptent les cadres procéduraux et ceux qui refusent de s'y laisser enfermer ${ }^{37}$.

19 La focalisation des travaux de sociologie politique sur les procédures de débat et de concertation a donné lieu à des critiques sévères, fondées sur une double absence : l'ensemble des scènes d'action et d'argumentation qui échappent au contrôle des organisateurs; la question des effets, des conséquences pratiques de la mise en débat ${ }^{38}$. Les problèmes de rapport aux institutions ne se posent pas de la même manière selon l'engagement des citoyens dans la composition des dispositifs. Une assemblée citoyenne qui émerge d'un mouvement social (e.g. l'assemblée des assemblées dans le cas des Gilets Jaunes ${ }^{39}$ ) ne traverse pas les mêmes épreuves de force et de légitimité que le panel des citoyens d'une conférence ou d'une convention citoyenne; une expérience d'énergie partagée sur un territoire donné n'a pas les mêmes contraintes qu'un collectif lancé dans une lutte contre une réforme nationale etc. Mais les tensions ne se réduisent pas à une question d'échelle. La tension la plus récurrente concerne l'opposition entre une quête d'autonomie et un travail d'inscription dans le jeu institutionnel ${ }^{40}$. L'autonomie visée par des collectifs d'acteurs, à l'instar des expériences menées en référence au municipalisme libertaire, n'est pas de même nature que le souci d'indépendance qui traverse les publics rassemblés par des dispositifs institutionnels autour d'une procédure et qui craignent avant tout des procédés d'influence et de manipulation, voire de renforcement d'un ordre hiérarchique ${ }^{41}$. Dans les expériences locales, le souci d'être reconnu par les autorités pour bénéficier de ressources provoque des frictions, entre les tenants d'un réalisme pratique et les porteurs d'expérimentations exigeant qu'elles soient reconnues comme telles, pour ce que les personnes et les groupes font réellement. Dans tous les cas, les acteurs qui tentent de faire bouger les lignes se heurtent aux cadrages qui leur sont imposés, souvent euxmêmes indexés sur des impératifs gestionnaires et administratifs, engageant des formes de conventionnement qui donnent la part belle aux acteurs déjà outillés et maitrisant les mondes bureaucratiques - et de fait peu enclins à remettre en cause l'ordre économique et politique. Dans le cas des dispositifs de consultation et de débat procéduralisés, c'est surtout le lien entre délibération et décision qui préoccupe les acteurs. Bref, sous les labels "participatif » et «citoyen » se déploient des logiques politiques différentes et souvent antagonistes. 
Un des points aveugles des multiples expériences participatives réside dans l'absence des relations de travail et des univers professionnels. Le fait que les dispositifs d'expression liés aux entreprises sont pris en charge par des entités collectives, organisations syndicales et autres instances d'expression et de représentation, rend difficile l'extension des publics concernés, instaurant un clivage problématique, souvent chez les mêmes personnes, entre travailleurs et citoyens. D'autant que les multiples mutations du travail ont éloigné de l'idéal de démocratie d'entreprise, partiellement mis en œuvre par les lois Auroux au début des années 1980, et qui ont fait long feu. Opposer les personnes en tant que citoyen-ne-s et en tant que salarié-es ou indépendant-e-s conduit à des impasses, pas seulement politiques mais aussi pour la circulation des savoirs et des manières de dire, de mettre en discussion des expériences - peut-on imaginer des salariés du nucléaire s'exprimer librement dans des arènes publiques $?^{42}$ L'éloignement des porteurs de la démocratie participative des sphères plus critiques, comme celles qui restent attachées au marxisme, a largement contribué à ce fossé, lequel a pour corollaire une fragmentation des mouvements de contestation et de critique sociale. Il faut ajouter au tableau la fracture, que plusieurs groupes d'acteurs tentent de réduire aujourd'hui, entre question sociale et question écologique. Il reste bien sûr l'univers des expérimentations coopératives portées par l'économie sociale et solidaire (ESS), très liée à l'ancrage d'alternatives économiques dans "les territoires ». L'ESS fait régulièrement l'objet de critiques. De nombreux acteurs-auteurs y voient une forme de localisme doublé d'une dépolitisation, souvent voilée par le recours aux mythes fondateurs des mouvements coopératifs historiques. S'y joue aussi une ambiguïté constitutive vis-à-vis de l'économie marchande: contre «la notion d'économie solidaire conçue comme un secteur particulier de l'économie ", il faudrait alors « réaffirmer la légitimité de l'extension des services collectifs non marchands " ${ }^{43}$.

21 Un des ressorts critiques les plus percutants des dispositifs institués de débat public et de démocratie participative tourne autour de la notion d'acceptabilité. Loin de permettre l'émergence de propositions alternatives ou de rendre justice aux contestations des publics convoqués, les dispositifs de participation auraient pour finalité de faire accepter les projets, en recourant à la figure classique de la supériorité de l'intérêt général sur la multiplicité des versions, des valeurs ou des options portées par les acteurs les plus divers. Le dispositif est conçu de telle manière que le porteur de projet, souvent appuyé par la puissance publique, semble disposer du monopole de la définition de l'intérêt général, modulo quelques aménagements à la marge, des ajustements ou des contraintes relatives à l'état du droit ${ }^{44}$. Cette critique de la participation domestiquée, souvent confortées par des observations empiriques ${ }^{45}$, embraye sur une autre qui concerne l'émergence de professionnels de la démocratie participative, consultants, animateurs de débats, experts en coproduction des savoirs, professionnels qui, de fil en aiguille, imposent ou contribuent à imposer des normes et des standards, contraignant les formes d'expression et les modalités d'enquête et d'argumentation auxquelles peuvent avoir recours les personnes ordinaires ${ }^{46}$. Il est notable qu'une des controverses les plus vives qui marquent ce champ de pratiques politiques, porte sur la légitimité du tirage au sort opposé aux collectifs organisés qui surgissent au fil des mobilisations et des conflits. 


\section{Entre délibération et conquête de prises sur le terrain : la bifurcation comme expérience collective}

Les travaux menés en sociologie pragmatique des alertes et des controverses n'ont cessé de mettre en évidence l'importance des moments d'épreuve dans lesquels l'espace des possible, et le sens commun de la réalité qui le sous-tend, fait l'objet d'un intense travail interprétatif collectif. Si les acteurs s'efforcent de réduire les doutes et les incertitudes, ils affrontent une indétermination radicale quant à ce qui adviendra dans le futur proche ou lointain. Le partage entre le réversible et l'irréversible se joue dans les moments d'intensification des échanges et des confrontations, dans des équilibres instables entre logique de discussion et conflictualitée ${ }^{47}$. Contrairement à la vision quelque peu manichéenne ou téléologique selon laquelle les acteurs dominants imposent toujours leurs projets et leurs dispositions, de multiples mobilisations, ainsi que des débats publics ou des conférences de citoyens, ont infléchi voire retourné un sens de l'histoire qui n'était pas nécessairement écrit ${ }^{48}$. Ces retournements ou ces réversibilisations n'ont pas d'effet immédiat sur des échelles plus globales ou « macro ", mais créent des précédents, inspirent d'autres scènes de débat et de lutte, et conduisent de plus en plus d'acteurs de terrain à faire des expériences, qu'ils transforment parfois en véritables expérimentations politiques.

Avoir prise sur les processus, c'est agir en amont des phénomènes d'irréversibilité, qu'il s'agisse de catastrophe, de transformation inéluctable ou de dépendance technopolitique: l'expérimentation au sens pragmatiste est ainsi une forme de laboratoire en monde ouvert où s'éprouvent et se réfléchissent les capacités d'action, et dans la foulée les conséquences de l'inaction, face aux ordres ou aux désordres qui viennent ou qui s'annoncent. Sans le développer ici, on peut mentionner le cas d'Europacity à Gonesse. Le conflit qui a opposé la coalition des promoteurs de ce giga centre commercial et des aménageurs de la Région Ile de France aux collectifs de riverains et de porteurs d'alternatives écologiques a tourné en faveur des derniers. Si le combat fut dur, il s'est caractérisé, comme l'a montré Stéphane Tonnelat, par un degré de violence bien moindre que d'autres conflits contre des grands projets inutiles imposés (on pense au conflit sur la ligne TAV Lyon-Turin, à Notre-Dame-des-Landes ou encore autour du projet Cigeo, nom du site d'enfouissement des déchets radioactifs à Bure). Tonnelat a montré l'importance, dans les différents moments du conflit du triangle de Gonesse, des expériences délibératives. Les protagonistes parviennent à lier par l'«intelligence collective" (notion deweyenne servant souvent de mythe fédérateur pour les nouveaux réformateurs sociaux), une forme de radicalité délibérément non-violente, même si la qualification de "violence " varie fortement d'un réseau d'acteurs à un autre, et une forme de pragmatisme, à travers un travail d'influence, de négociation et d'enrôlement en direction des univers professionnels et institutionnels, qui ne sont pas constitués comme un bloc ennemi uni et hostile par principe $^{49}$. Il s'agit donc aussi d'expérimenter, au fil de la lutte, les marges de manœuvre possibles au sein des organisations en créant de multiples allers-retours entre actions sur le terrain et démarches diplomatiques.

Dans toute expérience collective s'expriment des formes de tangibilité, des limites et des points d'arrêt, ces choses qui résistent dans l'expérience et que les philosophes pragmatistes, comme William James et John Dewey aident à penser. Mais la résistance du réel doit être éprouvée, et lorsqu'un désaccord survient, engage une mise en 
discussion, la convergence des expériences étant une des conditions de la production $\mathrm{du}$ tangible. Les expérimentations démocratiques saisies dans leur diversité contribuent ainsi au renouvellement des appuis du sens commun de la réalité et du sens critique qui le sous-tend. En ce sens, les épreuves collectives visant l'établissement d'une factualité, la portée de normes ou de règles, l'affirmation de biens ou de valeurs, ne doivent pas être découplées des approches critiques puisqu'elles ouvrent la voie au changement social - pour parler le langage des classiques de la sociologie. Alors que de simples écarts, de micro-variations sont généralement jugées inacceptables dans le système de référence dominant, la mise à l'épreuve, l'entrée dans un processus d'expérimentation permet l'émergence de nouveaux sujets et de nouveaux objets, l'invention de nouvelles prises collectives. Et c'est dans ce processus que naissent les alertes, les innovations ou les projets de transformation du monde que le pragmatisme sociologique s'attèle à prendre au sérieux, à l'ombre des doctrines hégémoniques.

\section{NOTES}

1. Jacques Rancière, La haine de la démocratie, Paris, La Fabrique, 2005; Bruno Bernardi, «L'opposition entre représentation et participation est-elle bien formée ? ", in La Vie des idées, 7 avril 2008, https://laviedesidees.fr/L-opposition-entre-representation.html

2. Pour une analyse sociologique des dispositifs expérimentaux en économie du développement, voir la thèse de Nassima Abdelghafour, Micropolitics of poverty: how randomized controlled trials address global poverty through the epistemic and political fragmentation of the world, Centre de Sociologie de l'Innovation, Université Paris-Sciences et Lettres, 2020.

3. Voir Antoine Hennion, "Enquêter sur nos attachements. Comment hériter de William James? ", in SociologieS, 23 février 2015, http://journals.openedition.org/sociologies/4953

4. Voir Joëlle Zask, «Pragmatisme et participation », in Ilaria Casillo et alii (dir.), Dictionnaire critique et interdisciplinaire de la participation, Paris, GIS Démocratie et Participation, bhttp:// www.dicopart.fr/fr/dico/pragmatisme-et-participation.

5. https://propositions.conventioncitoyennepourleclimat.fr/pdf/ccc-rapport-final.pdf

6. https://www.les150.fr/

7. D. Courant et Y. Sintomer observent une multiplication des usages du tirage au sort: "la sélection aléatoire peut désormais être revendiquée aussi bien par des autorités en place peu subversives que par des collectifs militant pour une démocratie radicale, par des think tanks libéraux que par des mouvements contestant les élites économiques, politiques et journalistiques » (Dimitri Courant et Yves Sintomer, «Le tirage au sort au XXIe siècle. Actualité de l'expérimentation démocratique ", in Participations, 2019/1, pp. 5-32.

8. Christophe Traïni, «L'Affaire du Siècle. Une mobilisation pour le climat à l'épreuve de la temporalité judiciaire», Texte de la communication au colloque Droit et temporalités, Paris, Sciences Po, avril 2021.

9. Voir Arnaud Gossement, «La convention citoyenne pour le climat est profondément monarchique » (entretien), in Reporterre, février 2020.

10. Voir sur ce point Daniel Cefaï et Daniel R. Huebner, «Pragmatisme et sociologie aux EtatsUnis. De Mead, Addams et Du Bois à l'interactionnisme symbolique », in Pragmata, 2, 2019. 
11. Voir sur ce point Francis Chateauraynaud et Josquin Debaz, Aux bords de l'irréversible. Sociologie pragmatique des transformations, Paris, Pétra, 2017.

12. Voir le volume spécial de la revue SociologieS publié en ligne 2015 et notamment l'introduction du dossier intitulé "Pragmatisme et sciences sociales: explorations, enquêtes, expérimentations » par Daniel Cefaï, Alexandra Bidet, Joan Stavo-Debauge, Roberto Frega, Antoine Hennion et Cédric Terzi

13. Ce qui là encore procède d'une longue histoire des mouvements sociaux. Le livre de référence est D. Cefaï, Pourquoi se mobilise-t-on ?, Paris, La Découverte, 2007.

14. Janine Barbot, Les Malades en mouvements. La médecine et la science à l'épreuve du sida, Paris, Balland, 2002,

15. Voir l'ouvrage collectif Didier Debaise et alii., Vie et Expérimentation. Peirce, James, Dewey, Paris, Vrin, 2008. Voir également, sur un mode plus analytique, l'usage par Sylvain Lavelle des travaux de Stanley Cavell, dans « Les philosophies ordinaires », in Revue philosophique de Louvain, 2021.

16. Yves Cohen, "Les foules raisonnables. Notes sur les mouvements sans parti ni leader des années 2010 et leur rapport avec le XXe siècle », in Politika, 2019 ; Laurent Jeanpierre, In Girum : les leçons politiques des ronds-points, Paris, La Découverte, 2019.

17. Paul Cossart et Julien Talpin, Lutte urbaine. Participation et démocratie d'interpellation à l'AlmaGare, Vulaine-sur-Seine, Éditions du Croquant, 2015.

18. Sur l'impératif de refonte des liens de la démarche analytique et de la pensée critique, à partir du pragmatisme, voir F. Chateauraynaud, "Social theory and the logic of inquiry. Some pragmatic arguments for a convergence of critical and reconstructive approaches ", in Alain Caillé \& Frédéric Vandenberghe, For a New Classic Sociology, Londres, Routledge, 2020.

19. Les analyses qui suivent tirent profit de plus d'une quinzaine d'années de séminaires à l'EHESS, à Paris et à Marseille, ainsi que des nombreux échanges au sein du Groupement d'Intérêt Scientifique (GIS) Démocratie\&Participation https://www.participation-et-democratie.fr/

20. Commission Nationale du Débat Public

21. Florian Charvolin, Les sciences participatives au secours de la biodiversité. Une approche sociologique, Paris, Rue d'Ulm, coll. « Sciences durables », 2019.

22. Daniel Cefaï, «La naissance de l'expérimentation démocratique. Quelques hypothèses de travail du pragmatisme ", in Pragmata, 2020, 3, pp. 270-355.

23. F. Chateauraynaud, Argumenter dans un champ de forces. Essai de balistique sociologique, Paris, Pétra, 2011.

24. Rien de tel que de grandes catastrophes pour réactiver les fondamentaux de ce que signifie être au monde, en faisant remonter tout à la fois des matrices communes et des singularités irréductibles. Voir Yoann Moreau, Vivre avec les catastrophes, Paris, Puf, 2017.

25. John Dewey, L'Art comme expérience (1934), traduit par Jean-Pierre Cometti, Paris, Gallimard, 2010.

26. F. Chateauraynaud, "Des disputes ordinaires à la violence politique. L'analyse des controverses et la sociologie des conflits", in La politisation :conflits et construction du politique depuis le Moyen Âge, Rennes, Presses universitaires de Rennes, 2010.

27. Voir Félix Tréguer, L'utopie déchue : une contre-histoire d'Internet XVe-XXIe siècle, Paris, Fayard, 2019.

28. Une preuve tangible est ce qui permet de faire converger des acteurs aux intérêts et représentations divergentes du fait de l'accessibilité de la mise à l'épreuve, rendant communes les prises qui permettent de tester la résistance des faits, voir F. Chateauraynaud, «L'épreuve du tangible ", in Bruno Karsenti et Louis Quéré (dir.), La Croyance et l'Enquête. Aux sources du pragmatisme, Paris, Éditions de l'EHESS, « Raisons pratiques », 2014.

29. J. Zask, «L'enquête sociale comme inter-objectivation », in B. Karsenti et L. Quéré (dir.), La Croyance et l'Enquête, op. cit., pp. 141-165. 
30. Sur la notion de « prise » comme concept central du pragmatisme sociologique, voir Christian Bessy et F. Chateauraynaud, Experts et faussaires. Pour une sociologie de la perception (2 ${ }^{\text {nd }}$ édition), Paris, Pétra, 2014.

31. Elise Demeulenaere, «Les semences entre critique et expérience : les ressorts pratiques d'une contestation paysanne », in Revue d'Études en Agriculture et Environnement, 94(4), 2013, pp. 421-441. 32. Voir F. Chateauraynaud et J. Debaz, « Alternatives métrologiques. La critique des « solutions » numériques et la fabrique de prises collectives », à paraître dans Laurence Allard (dir), Ecologies mobiles, 2021.

33. L'usage du terme "profane » est souvent problématique, en particulier dans un ouvrage qui fait pourtant figure de bible pour les promoteurs de sciences participative et de forums hybrides : Michel Callon, Pierre Lascoumes et Yannick Barthe, Agir dans un monde incertain. Essai sur la démocratie technique, Paris, La Découverte, 2001.

34. C'est le cas dans certaines expériences décrites par L. Centemeri dans l'univers, là encore fort hétérogène, de la permaculture. Laura Centemeri, La permaculture ou l'art de réhabiter, Paris, Editions Quae, 2019.

35. J. Dewey, Logique. La théorie de l'enquête, Paris, Puf, 2006.

36. Voir de nouveau F. Chateauraynaud et J. Debaz, Aux bords de l'irréversible. Sociologie pragmatique des transformations, op. cit.

37. On retrouve la distinction proposée naguère entre débats d' « élevage » et débats « sauvages » par le regretté Laurent Mermet, «Épilogue. Débattre sans savoir pourquoi : la polychrésie du débat public appelle le pluralisme théorique de la part des chercheurs ", in Cécile Blatrix (éd.), Le débat public: une expérience française de démocratie participative, Paris, La Découverte, « Recherches ", 2007, p. 368-380.

38. Sur les discussions relatives à cette focalisation, peu ou prou identifié comme une promotion politique des dispositifs de participation institués, voir Loï Blondiaux et Jean-Michel Fourniau, «Un bilan des recherches sur la participation du public en démocratie : beaucoup de bruit pour rien?», in Participations, 2011/1, pp. 8-35 ; Guillaume Gourgues, Sandrine Rui, Sezin Topçu, «Gouvernementalité et participation. Lectures critiques », in Participations, 2013/2, pp. 5-33.

39. http://assembleedesassemblees.org/

40. Le conflit de Notre-Dame-des-Landes, énormément étudié, a contribué à la mise en scène d'un conflit entre une conception radicale de l'autonomie et une logique de promotion d'alternatives acceptables par des acteurs institués, conduisant à des négociations avec les autorités et les aménageurs. Voir l'analyse qu'en propose sylvaine Bulle, à partir d'une immersion ethnographique, dans Irréductibles. Enquête sur des milieux de vie. De Bure à Notre-Dame-des-Landes, Grenoble, UGA, 2020.

41. Voir l'entretien d'Arnaud Gossement, «La convention citoyenne pour le climat est profondément monarchique ", in Reporterre, 5 février 2020.

42. Il y a malgré tout eu des moments de prise de parole. Voir les travaux de Marie GhisMalfilatre, «Les travailleurs de l'atome dans l'opposition au nucléaire : savoirs syndicaux, contreexpertise et citoyenneté au travail », in Sociétés contemporaines, $n^{\circ} 119,2020 / 3$.

43. Jean-Marie Harribey, « L'économie sociale et solidaire, un appendice ou un faux-fuyant ? », in Mouvements, 2002/1, pp. 42-49.

44. Jérôme Boissonade, Remi Barbier, Tom Bauler, Marie-José Fortin, Yann Fournis, Frédérick Lemarchand et Emmanuel Raufflet, "Mettre à l'épreuve l'acceptabilité sociale ", in Vertigo - la revue électronique en sciences de l'environnement, Volume 16, Numéro 1, mai 2016, https:// journals.openedition.org/vertigo/17163.

45. Voir la thèse de L. Bourdier, Socio-anthropologie des énergies marines renouvelables en BasseNormandie: gouverner (par) l'alternative, Université de Caen, 2019. L'impératif de transition énergétique étant posé comme incontournable et conforme à l'intérêt général, la thèse montre à partir notamment de la procédure de débat public concernant l'installation d'éoliennes en mer, 
comment « les acteurs locaux, y compris critiques des projets, sont mobilisés par les entreprises, l'État, les collectivités, et les scientifiques à l'aide de dispositifs visant à construire l'acceptabilité sociale. Avec ces dispositifs d'acceptabilité, on n'assiste pas à une transformation des projets techniques vers l'intégration de dimensions sociales, mais davantage à la translation d'enjeux sociaux en termes techniques. »

46. On peut bien sûr analyser la démocratie participative comme un nouveau marché. Voir Alice Mazeaud et Magali Nonjon, Le marché de la démocratie participative, Vulaine-sur-Seine, Editions du Croquant, 2018.

47. Marcelo Dascal, « Dichotomies and Types of Debate », in Frans H. van Eemeren \& Bart Garssen (eds), Controversy and Confrontation, Amsterdam, John Benjamins, 2008.

48. Laury-Anne Cholez, «Grands Projets inutiles. La lutte paie: mine de rien, les écologistes remportent de nombreuses victoires », in Reporterre, 15 décembre 2020.

49. Stéphane Tonnelat, « Lutter pour l'espace public - à propos d'EuropaCity », in AOC, juin 2021.

\section{RÉSUMÉS}

L'article expose les approches du pragmatisme sociologique, entre références philosophiques pragmatistes et mobilisations collectives contemporaines.

\section{INDEX}

Index chronologique : XXIème siècle

Mots-clés : Dewey John, Pragmatisme, sociologie, enquête, mouvements, expertise Index géographique: Monde

\section{AUTEUR}

\section{FRANCIS CHATEAURAYNAUD}

Directeur d'études en sociologie à l'école des hautes études en sciences sociales 